\title{
USO Y VALOR DE LA INFORMACIÓN PERSONAL: UN ESCENARIO EN EVOLUCIÓN
}

\section{Use and value of personal information: An evolving scenario}

José-Luis Gómez-Barroso

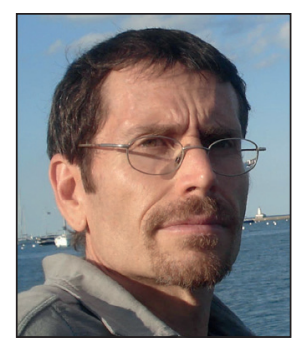

José-Luis Gómez-Barroso, profesor del Dpto. de Economía Aplicada e Historia Económica de la Univ. Nacional de Educación a Distancia (UNED), es doctor y licenciado en ciencias económicas y empresariales por la UNED, ingeniero de telecomunicación por la Univ. Politécnica de Madrid, y licenciado en derecho por la Univ. Complutense de Madrid. Trabaja en economía y regulación del sector convergente de las tecnologías de la información y de la comunicación, y en los factores que condicionan el desarrollo de la sociedad de la información. Es investigador principal del proyecto "Hacia un conocimiento de los mercados basados en el uso de información personal", financiado por el Ministerio de Economía y Competitividad a través del Programa estatal de investigación, desarrollo e innovación orientada a los retos de la sociedad (ECO2013-47055-R), en el que este artículo se inscribe.

http://orcid.org/0000-0001-9259-4915

Universidad Nacional de Educación a Distancia (UNED) Dpto. Economía Aplicada e Historia Económica Po Senda del Rey, 11. 28040 Madrid, España jlgomez@cee.uned.es

\section{Resumen}

Nuestras modernas sociedades de la información son también, y muy en particular, sociedades de la información personal. La colecta de datos personales se ha convertido en el activo necesario para el desarrollo de los mercados de la economía digital. Más importante aún, la manera en que los individuos perciben y gestionan la privacidad es ahora radicalmente distinta de la que era hace solo unos años. Aunque estas transformaciones sociales se han acelerado recientemente, el entorno en que debe contextualizarse el uso y valor dado a la información personal está en proceso de cambio desde hace ya medio siglo. Basándose en el análisis de la bibliografía académica que se ha ocupado del tema, este trabajo describe dicha evolución y analiza los intereses presentes de empresas, ciudadanos y administración pública en lo que a la revelación y uso de información personal se refiere.

\section{Palabras clave}

Información personal; Privacidad; Mercados digitales; Recolección de datos; Comportamiento racional; Datos masivos.

\begin{abstract}
Our modern information societies are also -and especially- personal information societies. The collection of personal data has become the key asset for the development of digital markets. Even more, the way in which now people perceive and manage their privacy totally contrasts with the situation just a few years ago. Although these social transformations have accelerated recently, the process of modification of the scenario contextualising the use and value of personal information is ongoing for half a century. By means of the analysis of academic literature on the topic, this article describes such an evolution as well as the current interests of companies, citizens and public administrations regarding the disclosure and use of personal information.
\end{abstract}

\section{Keywords}

Personal information; Privacy; Digital markets; Data collection; Rational behaviour; Big data.

Gómez-Barroso, José-Luis (2018). “Uso y valor de la información personal: un escenario en evolución”. El profesional de la información, v. 27, n. 1, pp. 5-18. 


\section{Introducción: público vs. privado}

Trazar los límites entre qué sea lo público y qué lo privado ha sido una preocupación fundamental y constante para diversas ramas del pensamiento humano ya desde la antigüedad. La definición de ambos términos no es unívoca y de hecho han sido y son empleados con diferentes sentidos en diferentes contextos (véase un repaso en Weintraub, 1997, en Steinberger, 1999 o en Krotoszynski, 2013). En consonancia con lo anterior, el Diccionario de la Lengua Española no sólo recoge varias acepciones para ambas voces, sino que las ha venido modificando. Es conveniente confrontar algunas de estas acepciones.

En acepción en desuso, público se dice de la potestad, jurisdicción y autoridad para hacer algo, como contrapuesto a privado, que sería aquí, de entre todas las acepciones posibles, lo particular y personal de cada individuo. A grandes rasgos, y con muchas matizaciones, ésta es la definición que funda la, frecuentemente utilizada pero no siempre evidente, separación entre derecho público y derecho privado. El derecho público regula (aparte de las relaciones de los Estados entre sí) las relaciones del Estado con sus ciudadanos, relaciones reguladas por normas de corte imperativo a diferencia del derecho privado en que prima la autonomía de voluntad de las partes a las que se supone en posición de igualdad.

Público es también lo perteneciente o relativo al Estado o a otra administración. Y su antónimo privado sería en este caso lo que no es de propiedad pública o estatal, sino que pertenece a particulares. Ésta es la línea de división que ha interesado básicamente a la economía: qué se deja al mercado y qué se reserva a la actividad pública es la primera pregunta a que hay que responder en la organización de la actividad económica.

Qué se deja al mercado y qué se reserva a la actividad pública es la primera pregunta a que hay que responder en la organización de la actividad económica

Por último, público es lo conocido o sabido por todos mientras que privado sería lo que se ejecuta a vista de pocos, familiar y domésticamente, sin formalidad ni ceremonia alguna. Esta acepción de privado, ligada con el concepto "intimidad" (zona espiritual íntima y reservada de una persona o de un grupo, especialmente de una familia), es la que resuena en la definición de "privacidad", que para el citado Diccionario es el ámbito de la vida privada que se tiene derecho a proteger de cualquier intromisión. Lo privado en este último sentido sería, paradójicamente, materia de derecho público, pues como la propia definición del Diccionario ya apunta, existe un interés público en su protección. Y además quedaría al margen de cualquier consideración económica pues no es concebible que pueda negociarse con un derecho básico.

Con este sentido las sucesivas regulaciones de lo privado (del ámbito privado) han seguido esta orientación y se han limita- do a establecer las barreras de la esfera personal que de ningún modo podían traspasarse. Un magnífico ejemplo lo constituye la redacción del artículo 18 de la Constitución española que, dentro de la categoría de derechos fundamentales, garantiza de modo genérico el derecho a la intimidad personal y familiar y en particular al secreto de las comunicaciones (en especial, de las postales, telegráficas y telefónicas). Además, en un previsor pero comprensiblemente poco concreto apartado 4 -dado el momento de su redacción-, establece que la ley limitará el uso de la informática para garantizar el honor y la intimidad personal y familiar de los ciudadanos y el pleno ejercicio de sus derechos. Los ponentes de la Carta Magna ya entreveían que algo estaba cambiando la informática pero con seguridad no eran conscientes (no podían serlo) de la magnitud del cambio. En una sociedad cada vez más mediada por relaciones computarizadas los peligros efectivamente se han multiplicado, y los esfuerzos de protección deben ser renovados y adaptados.

\section{En una sociedad cada vez más mediada por relaciones computarizadas los peli- gros se han multiplicado, y los esfuerzos de protección deben ser renovados y adaptados}

Conceptualmente es ésta una vía fácil de recorrer pues nada cambia en el entramado teórico descrito. Pero el cambio es mucho más profundo porque es también cualitativo. En apenas unos años, la convención social acerca de qué es lo que se ejecuta a vista de pocos, familiar y domésticamente, ha cambiado y sigue en acelerado proceso de cambio. Fragmentos de lo que es particular y personal de cada individuo se exponen sin pudor a la vista de familiares, amigos $y$, en ocasiones, a la vista de cualquiera. Datos personales se están cediendo a otros, a veces sin conocimiento del acto pero en muchas ocasiones con plena consciencia de lo que se hace, es decir, con plena autonomía de voluntad. Y en muchas ocasiones se trata de cesiones a cambio de un servicio, es decir de transacciones con sentido económico. Con todo el sentido económico pues, aunque no se utilice dinero y ni siquiera se dé un valor monetario a lo transferido, el intercambio de algo que pertenece a particulares también crea un mercado.

El fenómeno ha alcanzado tal magnitud que puede afirmarse que, voluntariamente revelada o no, la información personal constituye un componente fundamental del entramado de la moderna sociedad de la información. Ello hace que el estudio del uso y valor de la información personal, que tradicionalmente había venido interesando básicamente a la Economía y al Derecho, se convierta en materia de interés para casi cualquier ciencia social. Las aproximaciones que se pueden realizar son de hecho múltiples y en los próximos años se asistirá (se está asistiendo ya) a una multiplicación del número de trabajos provenientes de muy diferentes disciplinas. Sea cual sea la perspectiva adoptada, la descripción del statu quo es el necesario punto de partida. Este es el principal objetivo de este artículo, que pretende desmenuzar el escenario en que actualmente la información perso- 
nal se recopila y se utiliza, mirando antes hacia atrás para conocer el camino por el que se ha llegado a la situación presente. Sin aspirar a hacer una revisión bibliográfica exhaustiva, lo hace basándose en buena medida en la identificación y análisis de aquellos aspectos que han merecido en cada momento la atención de la doctrina académica. Cuáles sean esos aspectos en los años por venir ocupa la sección última de este trabajo.

La información personal constituye un componente fundamental del entramado de la moderna sociedad de la información

\section{Valor económico y regulación de la información personal: un repaso histórico}

\subsection{Un escenario estable: hasta mediada la década de los sesenta}

La regulación de la privacidad llevó durante siglos una existencia placentera pues el interés digno de protección jurídica se mantuvo básicamente inalterado. Se refería de modo genérico a la intimidad en la vida personal y familiar (Hafetz, 2002) y al secreto de las comunicaciones (véase el antiquísimo Belin, 1931). En la práctica, los tribunales, desde época tan temprana como el siglo XIV, lidiaron principalmente con escuchas o con violaciones de correspondencia (Holvast, 2009). El repaso de la bibliografía académica indica que tras la Segunda Guerra Mundial estaban apareciendo otras formas "modernas" de intrusión, tales como la apropiación de nombres o marcas o el acoso a los personajes públicos (Prosser, 1960), o también la revelación de historiales médicos (Coleman, 1961), pero sin que ello tuviera reflejo en los ordenamientos.

Desde los inicios del siglo XX, la noción legal se había ido consolidando, siempre con el contenido inicial, y así se recoge en el artículo 12 de la Declaración universal de derechos humanos (Naciones Unidas, 10 de diciembre de 1948):

"nadie será objeto de injerencias arbitrarias en su vida privada, su familia, su domicilio o su correspondencia, ni de ataques a su honra o a su reputación".

Similar es la redacción del artículo 8 de la Convención europea de derechos humanos (Consejo de Europa, 4 de noviembre de 1950) y también el del artículo 12 del posterior Pacto internacional de derechos civiles y políticos (Naciones Unidas, 16 de diciembre de 1966) así como el de muchas otras normas publicadas con posterioridad (como se vio en la introducción, la Constitución española de 1978 recoge idéntica definición). Sin embargo, en el momento de la firma del Pacto, a mediados de la década de los sesenta, el entorno, como se verá en el próximo subapartado, ya estaba alterándose.

Hasta ese momento no se había vinculado directamente información personal y negocio. Lo que no significa que este vínculo no existiera. Ya desde que se estableció la primera relación comercial, la información del otro ha tenido valor económico. Siempre como ahora, la confianza en que la otra parte cumpliría su parte en un pacto, y por tanto la probabilidad de que el pacto se cerrara, estaba condicionada por el conocimiento que del vendedor o del comprador se tuviera, particularmente si la transacción no se completaba en el momento del acuerdo o si la mercancía no era de fácil valoración. En las relaciones consolidadas (piénsese en un tendero de cualquier época), el conocimiento previo de los gustos del cliente ha simplificado la transacción y el conocimiento de sus circunstancias personales ha ayudado a tomar decisiones tan importantes como pudieran ser el fiarle o no.

De hecho no es completamente cierto que nadie hubiera reflexionado sobre el valor económico de la información personal. El progreso de la mercadotecnia como ciencia sí estaba creando una cierta consciencia, siquiera difusa, de su valor. La mercadotecnia estaba construyendo, en primer lugar, el marco teórico en que encajar algunas de las lecciones que los comerciantes habían aprendido (y utilizado) espontáneamente. En segundo lugar, estaba desarrollando nuevas teorías acerca de lo que era posible realizar disponiendo de datos de la clientela potencial. Esto se produjo muy en particular a partir de la segunda mitad del siglo XX, momento en que la producción industrial de bienes creció en tal cuantía que se comprendió que sólo conociendo las características de la demanda potencial podría venderse el producto. Conceptos como el de segmentación de mercados, con la consecuente adaptación del propio producto o del precio, pero muy en especial de la publicidad, se desarrollaron en la década del los cincuenta (Smith, 1956) y su práctica se fue difundiendo hasta ser hoy estrategias absolutamente comunes. Obviamente, la segmentación parte del conocimiento de las circunstancias sociales, económicas, geográficas y culturales de los posibles compradores, conocimiento destilado del proceso de los datos obtenidos, en aquel tiempo, mediante observación directa y mediante encuestas.

La segmentación del mercado parte del conocimiento de las circunstancias sociales, económicas, geográficas y culturales de los posibles compradores

\subsection{Un escenario en transformación: de la década de los sesenta hasta finales de la de los ochenta}

Como se ha dicho en el apartado anterior, el fin de la década de los sesenta puede tomarse como el momento en que se retoma la reflexión acerca de la naturaleza y alcance de la esfera personal. Una de las mejores constataciones de esa nueva inquietud la representa la obra de Alan Westin, quien en 1967 escribió que

"pocos valores tan fundamentales para la sociedad como lo es la privacidad han sido dejados en tal estado de indefinición por la teoría social o han sido sujeto de tan vaga y confusa redacción por los científicos sociales" (Westin, 1967). 
Westin trató, además, de dar perfiles nuevos y más nítidos al concepto de privacidad que para él

"es la reivindicación de personas, grupos o instituciones de poder determinar por sí mismos cuándo, cómo y con qué límites es comunicada a los demás información referida a ellos mismos".

Estas reflexiones no habían nacido empero espontáneamente, sino que eran consecuencia de los movimientos que estaban ocurriendo a su alrededor. De hecho, pese a que Westin recoge con razón buena parte del reconocimiento, otros coetáneos habían también avanzado por caminos convergentes con el suyo. Ya Bates (1964) abogaba por explorar el concepto de privacidad, lo que ayudaría a

"aclarar su valor potencial en el análisis de muchos problemas de psicología social y sociología".

Esfuerzos en esta línea y en esta época fueron también los de Jourard (1966), Bennett (1967) o Gross (1967).

La causa que está detrás de este casi repentino interés en entender qué implica lo privado es, como se ha dicho, el cambio de entorno. Cambio de entorno ante todo tecnológico (en su libro Westin dedica un capítulo a "El ordenador y la privacidad"). Las técnicas para el almacenamiento de información habían progresado y comenzaban a utilizarse con frecuencia por instituciones públicas y privadas. El uso que pudiera darse a estos almacenes de datos resultaba incierto. En su reseña de un libro de Edward Engberg, Ryan (1967) se mostraba indignado por dos decisiones del gobierno federal estadounidense: la obligación de rellenar, so pena de sanción, "largos y detallados" cuestionarios para el censo de 1970 y el proyecto de crear una base de datos centralizada y computarizada que iba a contener "la más sensible información personal”. También se percibía como una amenaza la rápida mejora de aparatos como cámaras o micrófonos, básicamente por las opciones de vigilancia y espionaje que ofrecían. Avanzando por esta dirección, el trabajo precursor de Michael (1964) ya había aventurado que

“[para 1984] compañías y gobiernos usarán los extraordinarios avances en la tecnología de computadores para recopilar y archivar hechos 'personales' [las comillas son del autor] de los ciudadanos e incluso para telecontrolar la población",

y algo más tarde Kalven (1967) definía un escenario prospectivo para final de siglo en que pronosticaba que todos viviríamos con un

"imborrable registro de nuestro pasado y nuestras limitaciones" con lo que la sociedad habrá perdido "su benévola capacidad de olvidar".

Conclusión clarividente. Como clarividente es el título del breve comentario de Foster (1968):

“los aparatos electrónicos pueden invadir la privacidad pero también protegerla".

Y como clarividente, aunque ingenua, es la conclusión del único trabajo que ligó en aquella época privacidad y mercadotecnia:

"la ley es sólo el marco de actuación para los profesionales de la investigación de mercados, y no supondrá una barrera mientras no se publiquen datos individuales; es la percepción de la profesión de hasta dónde llega el derecho a la privacidad lo que marca la auténtica barrera de lo que es legítimo" (Mayer; White, 1969).

Lo que sobre todo nos interesa aquí es constatar que para entonces la recopilación de datos era ya práctica difundida. De hecho, el telemarketing, es decir, el contacto directo con el cliente por teléfono era en aquellos años un fenómeno que comenzaba a ser habitual.

En la década de los setenta no se asistió a grandes progresos conceptuales pero sí se pudo observar un salto cuantitativo en el registro computarizado de actividades y transacciones, en el uso de estrategias más próximas al cliente o en el número de litigios en que se denunciaba una invasión de la intimidad. Varios países establecieron comités con el encargo de estudiar el fenómeno; en algunas ocasiones, las conclusiones a que llegaron se incorporaron a normas (una descripción detallada se encuentra en OCDE, 2011). A la vez, el mundo académico seguía tratando de acotar definiciones y riesgos, cada vez más patentes (como ejemplo Breckenridge, 1970; Godwin; Bode, 1971; Lusky, 1972; Goldstein; Nolan, 1975; Laufer; Wolfe, 1977). Y es que el volumen de datos que se guardaba no sólo había crecido exponencialmente, sino que, más importante, había comenzado a moverse a una velocidad creciente. Por ello, la $O C D E$ inició en 1978 el trabajo que culminó con la adopción de las "Directrices sobre protección de la privacidad y flujos transfronterizos de datos personales" en septiembre de 1980. Las directrices son casi simultáneas de la "Convención para la protección de las personas en lo relativo al procesamiento automático de datos personales", que el Consejo de Europa acordó también a finales de 1980 aunque el proceso de ratificación no comenzó hasta el año siguiente. En las directrices se definen los datos personales como

"cualquier información relacionada con un individuo identificado o identificable (sujeto de los datos)"

y se dedica un apartado a una serie de principios básicos en el manejo de la información personal que aún hoy siguen siendo la base de prácticamente todos los ordenamientos:

- finalidad legítima (especificación del propósito);

- proporcionalidad (limitación de recogida y uso);

- necesidad de consentimiento;

- transparencia (derecho al acceso y a la rectificación);

- responsabilidad (deber de custodia); y

- restricciones a la transmisión.

La publicación de las directrices de la OCDE también coincidió en el tiempo con la primera reflexión estructurada acerca del valor económico de la información personal. La Chicago School of Economics puede considerarse como precursora del análisis económico de la privacidad. Esta escuela mantenía que la privacidad tenía un efecto negativo en los mercados porque podría detener el flujo de la información privada, flujo que, a la postre, conduciría a una mejora de los niveles de actividad económica (Posner, 1978, 1981; Gould, 1980). Stigler (1980) defendía que cualquier interferencia pública en los mercados en que se utiliza información personal sería ineficiente desde el punto de vista económico: aquellos que amparándose en la regulación se negaran 
a revelar datos estarían ocultando información importante para el correcto funcionamiento de los mercados. Estas reflexiones crearon un limitado pero hasta entonces inexistente debate y algunas réplicas fueron inmediatas. Hirshleifer (1980) argumentaba que la racionalidad del individuo, asumida en el modelo de los miembros de la Chicago School, es un condicionante demasiado restrictivo al dejar de lado la complejidad de los procesos de decisión individuales.

\subsection{Consolidación de la transformación: las dos últi- mas décadas del siglo XX}

Desde el punto de vista normativo, las dos últimas décadas del siglo XX están marcadas por la consolidación y difusión de las directrices publicadas por la OCDE. La protección de los datos de carácter personal tomó cuerpo como derecho separado y distinto del respeto de la vida familiar y personal y de la protección de las comunicaciones. Esta diferenciación aparece en la mayor parte de las normas que se publicaron desde entonces. Valga por todas, la Carta de los derechos fundamentales de la Unión Europea, de marzo de 2000, cuyos artículos 7 y 8 tienen por título exactamente los conceptos que se acaban de citar. El primero de estos artículos sigue la línea tradicional, mientras que el segundo se ocupa de que toda persona tenga derecho a la protección de los datos de carácter personal que la conciernan, datos que

"se tratarán de modo leal, para fines concretos y sobre la base del consentimiento de la persona afectada o en virtud de otro fundamento legítimo previsto por la ley; toda persona tiene derecho a acceder a los datos recogidos que la conciernan y a su rectificación".

Las tarjetas de fidelización permiten registrar automáticamente todos los detalles de cada nueva compra del cliente

Podría considerarse el primero de los dos derechos recogidos en la Carta, el referido a la vida familiar, como derecho a la intimidad y el segundo como derecho a la privacidad. Sin embargo, el Diccionario Panhispánico de Dudas pone precisamente al derecho como ejemplo de los casos en que la distinción no debe hacerse ([Privacidad] no es sinónimo de intimidad, aunque ambos términos están semánticamente muy próximos y son intercambiables en algunos contextos: derecho a la intimidad, derecho a la privacidad).

Antes de la Carta de derechos fundamentales, la ya entonces Unión Europea había publicado, el 24 de octubre de 1995, la directiva relativa a la protección de las personas físicas en lo que respecta al tratamiento de datos personales y a la libre circulación de estos datos. Los datos personales son definidos con más detalle que en las directrices de la OCDE:

"toda información sobre una persona física identificada o identificable; se considerará identificable toda persona cuya identidad pueda determinarse, directa o indirectamente, en particular mediante un número de identificación o uno o varios elementos específicos, característicos de su identidad física, fisiológica, psíquica, económica, cultural o social".
Estos cambios normativos se gestaron mientras se estaba produciendo un vertiginoso salto adelante en el aprovechamiento económico de la información personal. Este salto fue de la mano de la cotidianidad del uso de lo que ya para entonces se incluía bajo el término tecnologías de la información y de la comunicación. Hitos ahora ya considerados históricos se sucedieron con rapidez:

- al inicio de la década de los ochenta se lanzó al mercado el primer ordenador considerado "personal";

- al inicio de la de los noventa la telefonía móvil comenzó a ser asequible;

- hacia 1995, internet comenzó a salir de los centros avanzados de investigación.

Poco hay que decir sobre la difusión social y geográfica de estas tecnologías. Es una perogrullada pero las tecnologías de la información sirven para el tratamiento de información, también, claro está, de la información personal. Las posibilidades para el almacenamiento y análisis de datos se fueron incrementando hasta desembocar en lo que desde casi fin de siglo se llama, por traducción directa del inglés, minería de datos (o "hallazgo de conocimiento en bases de datos"). A la par, la parte de comunicación de estas tecnologías lograba que las bases de datos se alimentaran en tiempo real con nuevos registros sin necesidad de intervención humana. Un buen ejemplo de estas opciones, aún hoy en boga, lo representan las tarjetas de fidelización que permiten registrar automáticamente todos los detalles de cada nueva compra del cliente. Estas tarjetas son conceptualmente interesantes por dos motivos:

- El primero es que permiten una interacción (publicidad, estrategia de venta) absolutamente personal con el cliente; es lo que se conoce como mercadotecnia relacional.

- El segundo es que son los propios clientes los que voluntariamente han cedido sus datos (en ocasiones han respondido a cuestionarios muy extensos) en el momento de incorporación al programa, lo que obviamente no está exento de riesgos desde el punto de vista de la privacidad (Davenport; Harris, 2007).

\section{El valor que muchas personas otorgan a su intimidad no parece excesivo si se compara con cuáles son los escasos be- neficios recibidos}

La causa de tal comportamiento es simple: la percepción de los beneficios de quien se inscribe (en forma de descuentos futuros $u$ otras recompensas) es superior al coste (desvelar sus datos). Pese a todas las puntualizaciones que se harán más tarde acerca de la no siempre clara percepción de cuáles sean esos costes, lo cierto es que esta decisión nos da pistas de que el valor que muchas personas otorgan a su intimidad (al menos a una parte de esa intimidad) no parece excesivo si se compara con cuáles son los beneficios recibidos.

Finalmente, desde el punto de vista del interés académico por la economía de la información personal, éste fue un período poco fértil. El debate de inicio de los ochenta no tuvo 
continuación y aparte de alguna reflexión en los inicios de los noventa sobre cómo manejar la privacidad en las actividades mercadotécnicas (Jones, 1991; Morris; Pharr, 1992; Foxman; Kilcoyne, 1993), no se puede destacar ninguna aportación hasta la de Gandy (1993), quien concluye que:

"su transformación [de la información personal] en una materia prima cuya producción, distribución y uso es gobernada por un conjunto de leyes, costumbres y restricciones tecnológicas genera una cantidad de problemas teóricos y prácticos que pone a prueba la imaginación de los economistas".

Ya algo más tarde, en 1996, Murphy (1996) confrontó las teorías utilitaristas de Chicago, poniendo al lado de los beneficios de revelar información los beneficios de ocultarla. Un año más tarde, una agencia del Department of Commerce de los Estados Unidos publicó un informe monográfico dedicado a la privacidad y a la autorregulación formado por un compendio de colaboraciones. En este informe, Varian (1997) retoma parte de los argumentos de la Chicago School y afirma que en ciertas ocasiones tendría un coste para los consumidores el que demasiado poca información acerca de ellos mismos fuera conocida. Con similares apoyos ideológicos, Noam (1997) afirma que la revelación u ocultación de la información se reduce a una cuestión de qué agente valora más esa información. Por último, Laudon (1997) propone el establecimiento de un mercado nacional de información en que los titulares de los datos podrían obtener un a modo de dividendo si quisieran vender los datos; básicamente es la teoría que ya había publicado un año antes (Laudon, 1996). En el filo del cambio de siglo, Samuelson (2000) analiza los pros y contras de que los usuarios negociaran libremente con sus datos personales.

\subsection{Un escenario incierto: el siglo XXI}

Si las décadas anteriores habían sido testigo de una auténtica convulsión en el uso de información personal, lo que ha traído la generalización del uso de internet ha sido un seísmo. En la Red la colecta de datos es realizada a una escala de otra magnitud y de forma inmediata y mucho menos costosa. Por el solo hecho de comunicarnos y de acceder a o intercambiar información dejamos trazas de quiénes somos, dónde estamos, qué hacemos o qué nos interesa, trazas que pueden ser (son de hecho) recogidas. Más aún, los datos personales son intercambiados o transferidos entre compañías e industrias, sin barreras geográficas ni de ningún otro tipo. Este proceso ha dado origen, como rasgo más representativo, al nacimiento de un tipo de empresas que obtiene directamente sus ingresos de la explotación de los datos personales. Empresas que en muchos casos no han parado de crecer y que forman el núcleo duro de la llamada economía de la información y, por ende, de la economía basada en la constante innovación. Aunque la frase resulte grandilocuente, es significativo que la (hasta 2010) comisaria europea para la protección de los consumidores, afirmara en un discurso que la información personal se está convirtiendo en el

"combustible de internet y la nueva moneda del mundo digital" (Kuneva, 2009).

En este siglo, las aproximaciones económicas a la privacidad provenientes de la academia se han multiplicado y en los últimos años el número de publicaciones sigue creciendo. Entre estos trabajos se incluyen conexiones de la situación actual con el debate iniciado hace más de treinta años sobre la eficiencia económica de la privacidad (Hermalin; Katz, 2006; Acquisti, 2009), reflexiones sobre el difícil equilibrio entre los intereses de las empresas y los de los individuos (Christiansen, 2011; Erramilli, 2012), repasos de bibliografía sobre el equilibrio entre publicidad y privacidad (Tucker, 2012) o sobre la privacidad en general (Pavlou, 2011), e incluso formalización de los mercados en que se intercambian datos personales haciendo uso de modelos microeconómicos (Rust et al., 2002; Taylor, 2004; Chellappa; Shivendu, 2006, 2008, 2010).

La siguiente frontera es progresar en el conocimiento cuantitativo del fenómeno de la utilización de datos personales (Gómez-Barroso; Feijóo, 2013). Algunos cálculos pueden encontrarse en Goldfarb y Tucker (2011), que aventuran que la regulación europea, más restrictiva que la estadounidense, reduce la efectividad de los anuncios en un 65\% (al no poder ser tan individualizados), o en Feijóo et al. (2014), quienes realizan una aproximación al valor de los datos partiendo de la información contable de algunas empresas. Pero el mayor desconocimiento es el que afecta a las operaciones que se producen en los mercados que involucran información personal. Qué datos son más valorados, cuánto se incrementa la rentabilidad de un anuncio por el hecho de dirigirse a una audiencia potencialmente más interesada, qué o cuánta información se cede a terceros y a qué precio; en definitiva, cómo exactamente los datos de los clientes generan beneficio, son cuestiones celosamente guardadas por las compañías. Nada ha trascendido y poco se ha investigado aún. Cauwels y Sornette (2012) afirman que los ingresos y beneficios de las compañías dedicadas a construir una red social están ligados con su base de usuarios

"mediante un canal directo que no tiene equivalente en otros sectores".

Pero es obvio que el estudio de estos fenómenos va muy por detrás de lo que está ocurriendo en los mercados y que son muchos aún los retos por abordar (Spiekermann et al., 2015).

La importancia presente de los flujos de información personal es tal que también ha interesado a otras disciplinas del ámbito de las ciencias sociales. De hecho estos flujos se han analizado desde una perspectiva política (Subramanian; Katz, 2011), perspectiva también empleada para estudiar la "soberanía de los datos" y el "emergente nacionalismo de la información" (Morozov, 2012) o los riesgos asociados con la "mercantilización de la privacidad" (Peekhaus, 2007). Los aspectos éticos son un nuevo e importante enfoque dado a los mercados de información personal (Hull, 2015), enfoque estrechamente ligado con el análisis de las bases filosófica (Michelfelder, 2010) y moral (Moore, 2010) de la privacidad. La psicología se ha interesado por determinar qué rasgos de la personalidad hacen más probable la revelación de datos (Liu et al., 2013; Osatuyi, 2015; Chen et al., 2016). En el campo de la comunicación también se han publicado contribuciones durante los últimos apenas diez años. Así, se ha estudiado con profusión la información revelada en foros virtuales (Humphreys et al., 2014; Min, 2016; More- 
no-Becerra et al., 2016) o la forma en que es gestionada (Crescenci et al., 2013; Külcü; Henkoğlu, 2014; Zhitomirsky-Geffet; Bratspiess, 2016) pero también se han analizado otros temas como el empoderamiento de los individuos en una sociedad impulsada por el uso de la información personal (Pierson, 2012). En esta línea, aunque Acquisti (2010) ya llamó la atención sobre el hecho de que

"vivimos una revolución comercial guiada por y enfocada a los datos de los consumidores en la que los individuos son a la vez consumidores y generadores de un activo realmente valioso: su información personal",

lo cierto es que el camino que conecta estas dos funciones no ha sido aún explorado. Por último, el fenómeno de los datos masivos o big data también ha añadido nuevos matices sea cual sea la disciplina involucrada (Leonard, 2014; Zhang, 2016; Gómez-Barroso et al., 2017).

\section{El nuevo escenario: los intereses en juego}

Tres son los actores que están presentes en la práctica totalidad de las actividades económicas: empresas, clientes y, con mayor o menor grado de involucramiento, pero al menos siempre estableciendo las reglas a que los intercambios deben someterse, los gobiernos. Son también los actores que intervienen en el ecosistema del intercambio de información personal, actuando como vértices del triángulo que forman los flujos de información.

\section{El modelo de negocio de muchos de los} gigantes de internet está basado exclusivamente en la explotación de datos personales con fines publicitarios

\subsection{Las empresas}

El interés de las empresas es claro: recopilar tantos datos como sea posible. Nada nuevo. Donde sí hay novedades es en el fin que se da a esos datos. La mayor parte de las empresas siguen explotándolos de un "modo tradicional", empleándolos como material con que desarrollar técnicas de mercadotecnia para comercializar sus propios productos. Pero ahora son posibles otras dos opciones:

-La primera es la utilización (o cesión) de los datos para lograr que la audiencia a la que llega la publicidad de un cierto producto sea mucho más interesante para el anunciante. No es un fenómeno nuevo pero sí mucho más relevante pues el modelo de negocio (los ingresos) de muchos de los gigantes de internet está basado exclusivamente en la explotación de datos personales con fines publicitarios. Piénsese en los portales de entrada (de facto los buscadores) o las redes sociales más conocidas. O, alternativamente, piénsese en cuántos modelos alternativos a la publicidad, por ejemplo los intentos de los medios de comunicación generalistas para condicionar el acceso a la información al pago de una suscripción, han llegado a buen puerto.

-La segunda opción a que se hacía referencia es directamente traficar con datos personales. El número de empresas que venden en la Red diferentes paquetes de datos personales

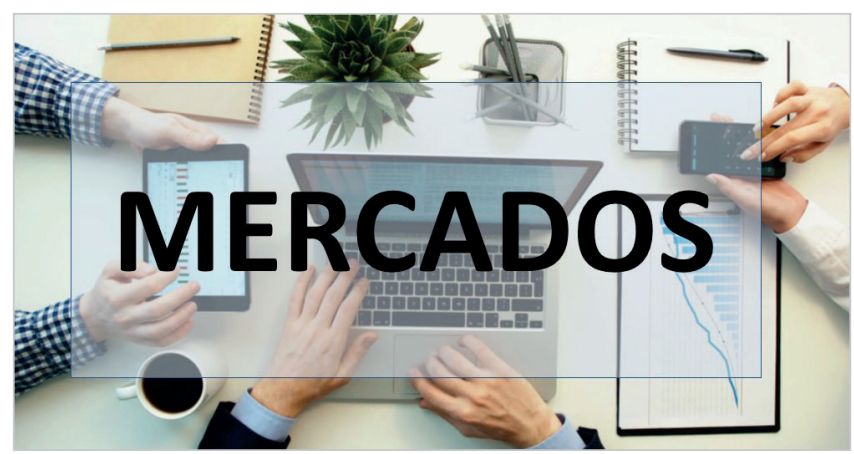

(desde simplemente el número de teléfono a un completo informe financiero) es considerable.

Los límites para la actividad de las empresas son dos: el primero el que viene marcado por las normas; el segundo el que marca su propio interés pues los clientes (al menos una parte de los clientes) no son insensibles ante estrategias agresivas y, por ejemplo, una publicidad demasiado personalizada puede resultar contraproducente (Okazaki et al., 2007; Peters et al., 2007).

\section{Las empresas buscan nuevas mañas para} que los usuarios desvelen más y más información

De no mediar cambios radicales en el entorno, la evolución futura a medio plazo no hará sino acentuar las tendencias que ahora ya se pueden observar. El valor (con seguridad creciente) de los datos personales hace que las empresas tengan incentivos incluso para bordear las normas y que busquen nuevas mañas para que los usuarios desvelen más y más información. De hecho, han acumulado conocimiento sobre cuáles son los parámetros de comportamiento de los usuarios y utilizan este conocimiento para promover la revelación de datos (Calo, 2014). Un ejemplo claro es la (supuesta) transparencia en la gestión de estos datos que muchas de las grandes compañías de internet han tomado recientemente por bandera, transparencia con la que se pretende aumentar la confianza y la percepción de control de los usuarios. El añadir el adjetivo "supuesta" viene del hecho de que, pese a lo prometido, las políticas de privacidad siguen siendo complicadas de descifrar incluso para los no profanos y las herramientas de control requieren más pasos de los que serían necesarios. De hecho, las aplicaciones tienen por lo general unos diseños en que el usuario necesita habilidades o conocimientos poco usuales para poder gestionar su privacidad. Considérese además que los contratos y licencias incorporan cláusulas leoninas que no pueden rechazarse o modularse pues el consentimiento o disentimiento se solicita para el conjunto.

A todo lo anterior cabe añadir que el uso de dispositivos móviles ha generado opciones de seguimiento aún más intenso, pues se añade la variable localización y además son artefactos con los que el usuario mantiene, en general, una relación más personal que con un ordenador de sobremesa, 
con lo que ello implica respecto a la información guardada y a la actividad realizada (Feijóo et al., 2010). Por si fuera poco, surgirán sin duda nuevas opciones de recogida de información con la creciente utilización de identificadores biométricos y con la difusión de la internet de las cosas.

Con la utilización de identificadores biométricos y la difusión de la internet de las cosas surgirán nuevas opciones de recogida de información

\subsection{Los usuarios}

En buena parte de los discursos o políticas referidos a la privacidad, los usuarios parecen ser sujetos pasivos (y damnificados) en este juego. No es siempre así. Al igual que sus datos sirven para personalizar la publicidad, también sirven para personalizar aplicaciones y servicios, y hacerlos más útiles y atractivos (Awad; Krishnan, 2006; Hammock; Rubin, 2011; Aguirre et al., 2015). Ciertas características de las comunicaciones móviles (conocimiento de quién se está conectando y desde dónde lo está haciendo) son precisas para la construcción de "contenidos aumentados" que ofrecen indudable valor añadido a quien los disfruta. En otros casos el beneficio está ligado a una percepción de recompensa social o psicológica; las redes sociales son el mejor ejemplo de ello (Krasnova et al., 2010; Benson et al., 2015). Y además la publicidad, y en concreto la publicidad personalizada, sustenta, no se olvide, el modelo del todo gratis en internet. Expresado en términos económicos, los usuarios reciben una "utilidad" cuando desvelan sus datos.

Pero es obvio que la moneda tiene dos caras y que una "desutilidad" va siempre unida a la posible utilidad. Los usuarios desconocen para qué exactamente se utilizan los datos que desvelan. Lo que es peor, ni siquiera tienen idea de qué otros datos suyos puedan tener las compañías. Nada saben de cómo o quién cruza datos para obtener perfiles o cómo son utilizados luego. Nada saben de si esos perfiles son genéricos 0 , pese a las promesas de anonimizar los resultados, se vinculan, si no a un nombre, sí a una dirección IP o a una dirección de correo electrónico. En estas circunstancias, el peligro de invasión de la privacidad es más que

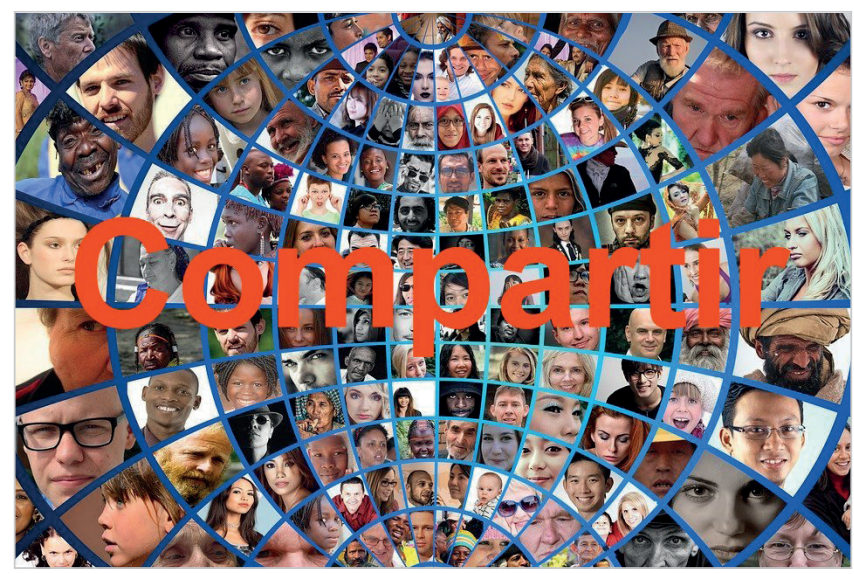

patente. Patente y real: si se hace el ejercicio sencillo de leer la política de privacidad de una compañía que opere en internet y se valora todo lo que se recopila, desasosiego es lo mínimo que puede sentirse. Si se lo propusiera y no debiera respetar límites legales, sería fácil para el buscador que utilizamos habitualmente (al que "preguntamos" sobre nuestras aficiones, proyectos, problemas, enfermedades...) saber de nosotros más que muchos de los que consideramos íntimos.

Un individuo racional en el sentido económico valoraría pros y contras y actuaría en consecuencia. ¿Pero son los usuarios de internet racionales cuando toman decisiones? La racionalidad necesita de información y la primera evidencia es que existe un amplio desconocimiento de los riesgos y de los medios para combatirlos. Muchas personas gestionan las situaciones que afectan su privacidad partiendo de malentendidos y confusiones acerca de las actividades de las empresas, las restricciones en el uso de los datos y la seguridad que se les ofrece, lo que les lleva a esperar mayores niveles de protección de los que realmente existen (Hoofnagle; Urban, 2014).

\section{Si se hace el ejercicio sencillo de leer la política de privacidad de una compañía que opere en internet y se valora todo lo que se recopila, desasosiego es lo míni- mo que puede sentirse}

¿Qué guía entonces el comportamiento de los individuos? Un conjunto de factores difícil de desentrañar, y con diferente peso en cada caso (Phelps et al., 2000; Heiman et al., 2013), entre los que está sin duda la percepción de lo que es socialmente aceptable (Trepte; Reinecke, 2013; Acquisti et al., 2012). En ese sentido, como el fenómeno de las redes sociales claramente demuestra, existe una tendencia creciente hacia la compartición de detalles de la vida privada. Pese a esta tendencia, el grado de inquietud con respecto a la propia intimidad es muy variado, lo que mezclado con el diferente grado de conocimiento de las posibles amenazas, hace que la percepción del grado de intrusismo que generan las prácticas habituales de las empresas sea variado (Wu et al., 2012; Schwaig, 2013) y que, desde luego, no pueda hablarse de un usuario tipo.

Un ejemplo de la falta de racionalidad de los individuos es la llamada paradoja de la privacidad, puesta de manifiesto hace años en otros contextos y confirmada en la actividad en internet por numerosos estudios (entre muchos otros Davis, 2003; Norberg et al., 2007; Young; Quan-Haase, 2013). La paradoja resulta de que aquello que los individuos responden en las encuestas (lo que dicen que harían) no coincide con la realidad (lo que realmente hacen). Niveles de privacidad que a priori se declaran inadmisibles son luego aceptados sin gran desasosiego en situaciones reales. Aunque la paradoja ha sido matizada en algunos estudios recientes (Baek, 2014; Dienlin; Trepte, 2015) lo que indica es que en realidad muchas personas no ven su intimidad como algo realmente valioso al menos en términos monetarios. Muchos de los que no estarían dispuestos a pagar 
nada, si se tratara de dinero, por acceder a una información o usar una aplicación, están dispuestos a rellenar cuestionarios de varias páginas para obtener esos servicios. Beresford et al. (2012) demuestran en un experimento la escasa disposición que existe a pagar por mantener ciertos niveles de privacidad; por ejemplo, la mayoría de encuestados estaban dispuestos a revelar cuáles eran sus ingresos si en la compra se les ofrecía un descuento de tan solo 1 euro. Jentzsch et al. (2012) o Strahilevitz y Kugler (2016) Ilegaron a resultados en la misma línea. Quizá sin ser plenamente conscientes de ello, muchos individuos han asumido (y aceptado) que el todo gratis va a costa de sus datos.

\subsection{El sector público}

Los gobiernos son los encargados de proteger los derechos de sus ciudadanos y, por tanto, los encargados de evitar prácticas que pongan en peligro su privacidad.

Pero su papel no es meramente el de policía autónomo ajeno a lo que ocurre. También es parte en el juego y la protección debe extenderse también a sus propias prácticas. No debe olvidarse que las administraciones son el actor que más cantidad de datos personales almacena. Por tanto, un primer deber que se deriva es el de custodia, que se hace más imperativo cuando los datos se ponen a disposición de terceros, lo que ocurre cada vez más por la aceptación de la filosofía de "datos accesibles" (en inglés open data, expresión utilizada con más frecuencia). Pero además hay que tener en cuenta que las administraciones públicas también confrontan datos de sus ciudadanos/contribuyentes/electores. Y de hecho una administración efectiva, que en la era de la comunicación instantánea no obligue a los administrados a ir de ventanilla en ventanilla, necesita del traspaso de datos entre diferentes órganos. Obviamente, por legítimos que sean los fines, estas actividades también deben estar regladas.

Más importante aún es el conflicto entre privacidad y seguridad. Como varias noticias recientes (más de la cuenta) demuestran, los gobiernos pueden ser también invasores de la privacidad. Incluso los más peligrosos invasores si nos atenemos al grado de control que se ha desvelado. Se trata de otro debate diferente pero obviamente ligado con el de la protección de datos. Ante el aumento de los controles por razones de seguridad, ¿dónde está el límite? ¿El fin justifica cualquier medio?

Paradoja de la privacidad: lo que los individuos responden en las encuestas (lo que dicen que harían) no coincide con la realidad (lo que realmente hacen)

Otra variable en la ecuación es el impulso de la economía digital. Esas empresas que se mantienen gracias a la recolección de datos (y que en muchos casos no han parado de crecer) forman el núcleo duro de esa economía de la innovación basada en la información que todos los gobiernos ven como una de las vías más estables para el crecimiento económico futuro. ¿Mantendrían los gobiernos el mismo discurso, o el mismo grado de severidad con respecto a la

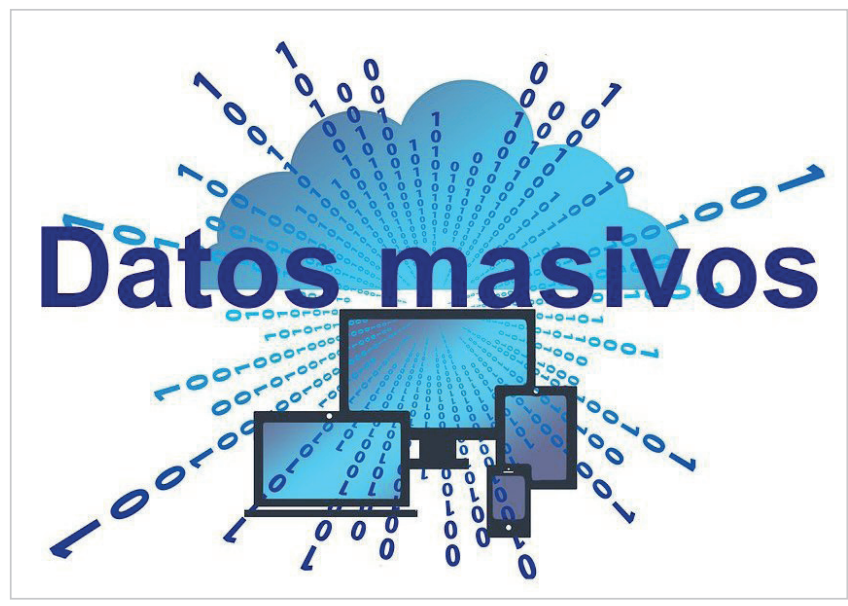

utilización de datos personales, si esas empresas estuvieran radicadas en su territorio? El dilema no es baladí pues una normativa demasiado estricta podría hacer que las empresas emigraran a otros lugares más permisivos desde donde seguirían dando servicio a los nacionales del primer país, que finalmente no estarían en una situación mejor que la inicial. La Red es global y es casi imposible poner puertas en internet. Lo que nos lleva al que quizá es el problema más serio que afronta el legislador: tratándose de la actividad más global de un mundo globalizado, las regulaciones son difíciles de hacer respetar si no son también globales.

La mayoría de encuestados estaban dispuestos a revelar cuáles eran sus ingresos si en la compra se les ofrecía un descuento de tan solo 1 euro

\section{Reflexión final: mirando al futuro}

La consideración de qué es y cómo se maneja lo privado es uno de los trazos más sobresalientes de la sociedad en cambio acelerado que caracteriza al nuevo siglo. Acciones de exposición a los demás que hoy son absolutamente comunes hubieran sido impensables hace solo unos cuantos años. Si entonces se hubiera preguntado si nos era posible imaginarnos adoptando dicho comportamiento, la contestación hubiera sido una nueva pregunta: ¿para qué?, ¿a quién le importa que yo...? Esta pregunta tiene hoy respuesta: le importa a nuestro círculo de próximos (al menos se comparte información muchas veces banal con la convicción de que sí les interesa) pero también a empresas y, dependiendo de la situación concreta, a desconocidos o incluso a los poderes públicos. Y lo más importante es que esta respuesta no causa la enorme desazón que hubiera generado en el pasado. Ciertamente, cuando las propias vidas son en ocasiones casi retransmitidas cabe pensar que la percepción de intrusión en la intimidad se ha alterado, lo que indudablemente influye en cómo se reacciona frente a las solicitudes de datos personales. Una mayor permisividad, incluso indiferencia, se mezcla en muchos casos con ignorancia acerca de lo que ocurre con nuestros datos y también de las vías posibles de protección. El resultado es que si alguien (un gran hermano) 
se encargara de juntar los datos voluntariamente revelados o cedidos a cambio de un servicio, con los recogidos sin consentimiento expreso aquí y allá, los ordenara y asociara con un nombre, la identidad y vida de una parte cada vez mayor de los habitantes del planeta podría ser retratada con bastante exactitud.

Ante el aumento de los controles por razones de seguridad, ¿dónde está el límite? ¿¿El fin justifica cualquier medio?

Sin necesidad de imaginar futuros alarmantes, lo cierto es que datos con alguna carga personal son una parte importante (y creciente) de los intercambios que se producen en la Red. Y puesto que allí se desarrolla cada día con más intensidad la actividad comercial, cultural, política y social, el uso y valor de la información personal seguirá atrayendo (cada vez más) la atención de toda disciplina del grupo de las ciencias sociales.

Comenzando con la Economía, se progresará en el conocimiento de los mercados basados en el uso de información personal y, en particular, de los modelos de negocio que la utilizan intensivamente. La mercadotecnia seguirá siendo un campo prioritario de desarrollo y experimentación. Parte de todo ese progreso se basará en una mejor comprensión de nuestro comportamiento en aquellas situaciones que implican cesión de datos, para lo que se aliará con la Psicología en diseños experimentales y formalización de modelos conductuales. El Derecho debe ayudar a orientar las (necesarias) reformas legales. El ordenamiento que regula la privacidad debe adaptarse a la nueva situación o perderá, si no su sentido, sí con seguridad su eficacia. Y es que aunque la privacidad es desde luego un valor jurídico digno de protección (y ha de seguir siéndolo), la situación descrita es lo suficientemente concluyente como para admitir que son necesarias otras orientaciones que tengan en cuenta la realidad de lo que ocurre en internet. Más aún, para que unos usuarios ahora mayoritariamente desinformados, e incluso indolentes respecto al tema, sean capaces de ejercer su libre voluntad se necesita algo más que legislar. La voluntad debe estar capacitada y debe tener medios con que ejercerse o no será libre. Todo lo anterior hace pensar que la aproximación necesaria en un escenario del siglo XXI es más de política de privacidad que de estricta regulación de la privacidad. La regulación es una parte básica de esa política pero ha de ser completada con otras acciones cuyo fin último sea no la protección de los usuarios sino el dar a esos usuarios la opción a estar protegidos. La Educación y la Ciencia Política tienen algo que decir en este escenario. Ciencia Política que además tiene que seguir considerando prioritariamente las conexiones de una sociedad menos privada con una sociedad vigilada. Esos son los dos campos en donde más progreso cabe esperar por lo magro de sus aportaciones actuales. Siendo, como es, la revelación de información personal un fenómeno sociológico de primer orden, sorprende que apenas existan ahora trabajos que puedan incluirse en el campo de la Sociología. Por último, el campo de las Ciencias de la Información está necesitado de contribuciones que exploren, entre otras vías posibles, el uso de la información personal (pero pública) en los procesos generales de comunicación y en particular en la comunicación periodística, la explotación de datos en la industria de contenido, o la transformación del sistema publicitario.

Hecha esta separación por áreas, que es útil a efectos de clasificación, debe admitirse que son necesarios, y muy probablemente mucho más interesantes, enfoques comprehensivos. Como fenómeno social generalizado que es, incluso las visiones que pretendan caracterizar aspectos muy concretos del uso o gestión de la información personal deben probablemente considerar su incidencia en campos afines. Como ejemplo, una campaña de publicidad personalizada debe tener en cuenta los aspectos comunicativos, psicológicos o incluso sociológicos, pero también los económicos o los jurídicos que marcan los límites de lo factible.

Si un gran hermano se encargara de juntar los datos voluntariamente revelados o cedidos a cambio de un servicio, con los recogidos sin consentimiento expreso aquí y allá, la vida de mucha gente podría ser retratada con bastante exactitud

A más largo plazo, los retos variarán. Del mismo modo que ahora nos comportamos, siempre en relación con la barrera entre público y privado, de un modo que era difícil de prever hace sólo unos años, es muy probable que en el futuro aparezcan tendencias que ni siquiera imaginamos. Lo cierto es que en una sociedad de la información (también) personal, cómo se usan y cómo gestionamos nuestros datos seguirá siendo un tema sugerente para todo investigador que quiera contribuir a incrementar nuestro conocimiento de la sociedad en que vivimos.

\section{Agradecimientos}

Este artículo es un resultado del proyecto "Hacia un conocimiento de los mercados basados en el uso de información personal", financiado por el Ministerio de Economía y Competitividad a través del Programa estatal de investigación, desarrollo e innovación orientada a los retos de la sociedad (ECO2013-47055-R).

\section{Bibliografía}

Acquisti, Alessandro (2009). "Nudging privacy: The behavioral economics of personal information". IEEE security \& privacy, v. 7, n. 6, pp. 82-85.

https://doi.org/10.1109/msp.2009.163

Acquisti, Alessandro (2010). The economics of personal data and the economics of privacy. Joint WPISP-WPIE Roundtable "The economics of personal data and privacy: 30 Years after the OECD privacy guidelines". Background paper \#3. https://www.oecd.org/sti/ieconomy/46968784.pdf

Acquisti, Alessandro; John, Leslie K.; Loewenstein, George (2012). "The impact of relative standards on the propensity to disclose". Journal of marketing research, v. 49, n. 2, pp. 
160-174

https://doi.org/10.1509/jmr.09.0215

Aguirre, Elizabeth; Mahr, Dominik; Grewal, Dhruv; DeRuyter, Ko; Wetzels, Martin (2015). "Unraveling the personalization paradox: The effect of information collection and trust-building strategies on online advertisement effectiveness". Journal of retailing, v. 91, n. 1, pp. 34-49.

https://doi.org/10.1016/j.jretai.2014.09.005

Awad, Naveen-Farag; Krishnan, M. S. (2006). "The personalization privacy paradox: An empirical evaluation of information transparency and the willingness to be profiled online for personalization". MIS quarterly, v. 30, n. 1, pp. 13-28. http://www.jstor.org/stable/25148715

Baek, Young-Min (2014). "Solving the privacy paradox: A counter-argument experimental approach". Computers in human behavior, v. 38, pp. 33-42.

https://doi.org/10.1016/j.chb.2014.05.006

Bates, Alan P. (1964). "Privacy - A useful concept?". Social forces, v. 42, n. 4, pp. 429-434.

https://doi.org/10.2307/2574986

Bélanger, France; Crossler, Robert E. (2011). "Privacy in the digital age: a review of information privacy research in information systems". MIS quarterly, v. 35, n. 4, pp. 1017-1042. http://aisel.aisnet.org/misq/vol35/iss4/12

Belin, Édouard (1931). "The privacy of telegraphic and radiotelegraphic transmissions". Comptes rendus hebdomadaires des séances de l'Académie des Sciences, v. 193, pp. 25-27.

Bennett, Chester C. (1967). "What price privacy?". American psychologist, v. 22, n. 5, pp. 371-376.

https://doi.org/10.1037/h0024927

Benson, Vladlena; Saridakis, George; Tennakoon, Hemamaali (2015). "Information disclosure of social media users". Information technology \& people, v. 28, n. 3, 426-441.

https://doi.org/10.1108/itp-10-2014-0232

Beresford, Alastair R.; Kuebler, Dorothea; Preibusch, Sören (2012). "Unwillingness to pay for privacy: A field experiment". Economics letters, v. 117, n. 1, pp. 25-27.

https://doi.org/10.1016/j.econlet.2012.04.077

Breckenridge, Alan-Carlyle (1970). The right to privacy. Lincoln: University of Nebraska Press. ISBN: 978-0-8032-6228-7

Calo, Ryan (2014). "Digital market manipulation”. The George Washington law review, v. 82, 995, 57 pp.

https://doi.org/10.2139/ssrn.2309703

Camenisch, Jan (2012). "Information privacy?!". Computer networks, v. 56, n. 18, pp. 3834-3848.

https://doi.org/10.1016/j.comnet.2012.10.012

Cauwels, Peter; Sornette, Didier (2012). “Quis pendit ipsa pretia: Facebook valuation and diagnostic of a bubble based on nonlinear demographic dynamics". Journal of portfolio management, v. 38, n. 2, pp. 56-66.

https://doi.org/10.2139/ssrn.2191556

Chellappa, Ramnath K.; Shivendu, Shivendu (2006). “A model of advertiser-portal contracts: personalization strate- gies under privacy concerns". Information technology and management, v. 7, n. 1, pp. 7-19.

https://doi.org/10.1007/s10799-006-5726-9

Chellappa, Ramnath K.; Shivendu, Shivendu (2008). "An economic model of privacy: A property rights approach to regulatory framework". Journal of management information systems, v. 24, n. 3, pp. 193-225.

https://doi.org/10.2753/mis0742-1222240307

Chellappa, Ramnath K.; Shivendu, Shivendu (2010). "Mechanism design for 'free' but 'no free disposal' services: The economics of personalization under privacy concerns". Management science, v. 56, n. 10, pp. 1766-1780.

https://doi.org/10.1287/mnsc.1100.1210

Chen, Xi; Pan, Yin; Guo, Bin (2016). "The influence of personality traits and social networks on the self-disclosure behavior of social network site users". Internet research, v. 26, n. 3, pp. 566-586.

https://doi.org/10.1108/intr-05-2014-0145

Christiansen, Linda (2011). "Personal privacy and Internet marketing: An impossible conflict or a marriage made in heaven?". Business horizons, v. 54, n. 6, pp. 509-514. https://doi.org/10.1016/j.bushor.2011.06.002

Coleman, Arthur H. (1961). "The patient's right to privacy". Journal of the National Medical Association, v. 53, n. 2, p. 207. https://www.ncbi.nlm.nih.gov/pmc/articles/PMC2641881/ pdf/jnma00690-0108.pdf

Crescenzi, Lucrezi; Araüna, Núria; Tortajada, Iolanda (2013). "Privacy, self-disclosure and self-image of Spanish teenagers on social networking sites. The case of Fotolog". Comunicación y sociedad, v. 26, n. 2, pp. 65-78.

https://www.unav.es/fcom/communication-society/es/ articulo.php?art_id $=448$

Davenport, Thomas H.; Harris, Jeanne G. (2007). "The dark side of customer analytics". Harvard business review, v. 85, n. 5, pp. 37-43.

https://hbr.org/2007/05/the-dark-side-of-customer-analytics

Davis, Charles N. (2003). "Electronic access to information and the privacy paradox: Rethinking practical obscurity and its impact on electronic freedom of information". Social science computer review, v. 21, n. 1, pp. 15-25.

https://doi.org/10.1177/0894439302238968

Dienlin, Tobias; Trepte, Sabine (2015). "Is the privacy paradox a relic of the past? An in-depth analysis of privacy attitudes and privacy behaviors". European journal of social psychology, v. 45, n. 3, pp. 285-297.

https://doi.org/10.1002/ejsp.2049

Erramilli, Vijay (2012). "The tussle around online privacy". IEEE internet computing, v. 16, n. 4, pp. 69-71. https://doi.org/10.1109/mic.2012.92

Feijóo, Claudio; Gómez-Barroso, José-Luis; Martínez-Martínez, Inmaculada J. (2010). "Nuevas vías para la comunicación empresarial: publicidad en el móvil". El profesional de la información, v. 19, n. 2, pp. 140-148. https://doi.org/10.3145/epi.2010.mar.04

Feijóo, Claudio; Gómez-Barroso, José-Luis; Voigt, Peter 
(2014). "Exploring the economic value of personal information from firms' balance sheets". International journal of information management, v. 34, n. 2, pp. 248-256. https://doi.org/10.1016/j.ijinfomgt.2013.12.005

Foster, John E. (1968). "Electronic devices can protect privacy as well as invade it". Computers and automation, v. 17, n. 5, p. 15

https://goo.gl/G7akRS

Foxman, Ellen R.; Kilcoyne, Paula (1993). "Information technology, marketing practice, and consumer privacy - Ethical issues". Journal of public policy \& marketing, v. 12, n. 1 , pp. 106-119.

https://www.jstor.org/stable/30000116

Gandy, Oscar H. (1993). "Toward a political-economy of personal information". Critical studies in mass communication, v. 10, n. 1, pp. 70-97.

https://doi.org/10.1080/15295039309366849

Godwin, William F.; Bode, Katharine-Anne (1971). "Privacy and new technology". Personnel and guidance journal, v. 50, n. 4, pp. 298-304.

Goldfarb, Avi; Tucker, Catherine E. (2011). "Privacy regulation and online advertising". Management science, v. 57, n. 1, pp. 57-71.

https://doi.org/10.2139/ssrn.1600259

Goldstein, Robert C.; Nolan, Richard L. (1975). "Personal privacy versus corporate computer". Harvard business review, v. 53, n. 2, pp. 62-70.

Gómez-Barroso, José-Luis; Feijóo, Claudio (2013). "Información personal: La nueva moneda de la economía digital". El profesional de la información, v. 22, n. 4, pp. 290-297. https://doi.org/10.3145/epi.2013.jul.03

Gómez-Barroso, José-Luis; Feijóo, Claudio; Martínez, Dolores F. (2017). "Política antes que regulación: La protección de la información personal en la era del big data". Economía industrial, n. 405, pp. 113-119.

https://www.dykinson.com/libros/revista-economiaindustrial-numero-405/0422-2784-numero-405

Gould, John P. (1980). "Privacy and the economics of information". Journal of legal studies, v. 9, n. 4, pp. 827-842. http://www.journals.uchicago.edu/doi/abs/10.1086/467668

Gross, Hyman (1967). "Concept of privacy". New York University law review, v. 42, n. 1, pp. 34-54.

Hafetz, Jonathan L. (2002). "'A man's home is his castle?': Reflections on the home, the family, and privacy during the late nineteenth and early twentieth centuries". WiIliam \& Mary journal of women and the law, v. 8, n. 2, pp. 175-242.

http://scholarship.law.wm.edu/wmjowl/vol8/iss2/2

Hammock, Michael R.; Rubin, Paul H. (2011). "Applications want to be free: Privacy against information". Competition policy international, v. 7, n. 1, pp. 41-58.

https://goo.gl/2Huacc

Heirman, Wannes; Walrave, Michel; Ponnet, Koen (2013). “Predicting adolescents' disclosure of personal information in exchange for commercial incentives: An application of an extended theory of planned behavior". Cyberpsychology, behavior and social networking, v. 16, n. 2, pp. 81-87. https://doi.org/10.1089/cyber.2012.0041

Hermalin, Benjamin E.; Katz, Michael L. (2006). "Privacy, property rights and efficiency: The economics of privacy as secrecy". Quantitative marketing and economics, v. 4, n. 3, pp. 209-239. https://doi.org/10.1007/s11129-005-9004-7

Hirshleifer, Jack (1980). "Privacy - Its origin, function, and future". Journal of legal studies, v. 9, n. 4, pp. 649-664. https://doi.org/10.1086/467659

Hoofnagle, Chris J.; Urban, Jennifer M. (2014). "Alan Westin's privacy homo economicus". Wake Forest law review, v. 49 , n. 2, pp. 261-317.

https://papers.ssrn.com/sol3/papers.cfm?abstract $i d=2434800 \& \mathrm{rec}=1 \& \mathrm{srcabs}=2433912 \mathrm{a} \mathrm{alg}=1 \& p o s=6$

Hull, Gordon (2015). "Successful failure: what Foucault can teach us about privacy self-management in a world of Facebook and big data". Ethics and information technology, v. 17, n. 2, pp. 89-101.

https://doi.org/10.1007/s10676-015-9363-z

Humphreys, Lee; Gill, Phillipa; Krishnamurthy, Balachander (2014). "Twitter: a content analysis of personal information". Information communication \& society, v. 17, n. 7 , pp. 843-857.

https://doi.org/10.1080/1369118x.2013.848917

Jentzsch, Nicola; Preibusch, Sören; Harasser, Andreas (2012). Study on monetising privacy. An economic model for pricing personal information. Enisa deliverable - 2012-02-27. Heraklion: European Network and Information Security Agency. https://www.enisa.europa.eu/publications/monetising-privacy

Jones, Mary-Gardiner (1991). "Privacy: A significant marketing issue for the 1990s". Journal of public policy \& marketing, v. 10, n. 1, pp. 133-148.

https://goo.gl/suYw6Y

Jourard, Sidney M. (1966). "Some psychological aspects of privacy". Law and contemporary problems, v. 31, n. 2, pp. 307-318.

https://doi.org/10.2307/1190673

Kalven, Harry (1967). "The problems of privacy in the year 2000". Daedalus, v. 96, n. 3, pp. 876-882

Krasnova, Hanna; Spiekermann, Sarah; Koroleva, Ksenia; Hildebrand, Thomas (2010). "Online social networks: why we disclose". Journal of information technology, v. 25, n. 2, pp. 109-125.

https://doi.org/10.1057/jit.2010.6

Krotoszynski, Ronald J. (2013). "The polysemy of privacy". Indiana law journal, v. 88, n. 3, pp. 881-918. https://www.repository.law.indiana.edu/ilj/vol88/iss3/2

Külcü, Özgür; Henkoğlu, Türkay (2014). "Privacy in social networks: An analysis of Facebook". International journal of information management, v. 34, n. 6, pp. 761-769.

https://doi.org/10.1016/j.ijinfomgt.2014.07.006 
Laudon, Kenneth C. (1996). "Markets and privacy". Communications of the ACM, v. 39, n. 9, pp. 92-104.

https:// doi.org/10.1145/234215.234476

Laudon, Kenneth C. (1997). "Extensions to the theory of markets and privacy: Mechanics of pricing information". En: Wellbery, B. S. (ed.), Privacy and self-regulation in the information age, cap. 1-D. Washington: National Telecommunications and Information Administration.

http://www.ntia.doc.gov/page/chapter-1-theory-marketsand-privacy

Laufer, Robert S.; Wolfe, Maxine (1977). "Privacy as a concept and a social issue: A multidimensional developmental theory". Journal of social issues, v. 33, n. 3, pp. 22-42. https://dx.doi.org/10.1111/j.1540-4560.1977.tb01880.x

Leonard, Peter (2014). "Customer data analytics: privacy settings for 'Big Data' business". International data privacy law, v. 4, n. 1, pp. 53-68.

https://doi.org/10.1093/idpl/ipt032

Liu, Cong; Ang, Rebecca P.; Lwin, May O. (2013). "Cognitive, personality, and social factors associated with adolescents' online personal information disclosure". Journal of adolescence, v. 36, n. 4, pp. 629-638.

https://doi.org/10.1016/j.adolescence.2013.03.016

Lusky, Louis (1972). "Invasion of privacy - Clarification of concepts”. Political science quarterly, v. 87, n. 2, pp. 192-209. https://doi.org/10.2307/2147825

Mayer, Charles S.; White, Charles H. (1969). "The law of privacy and marketing research". Journal of marketing, v. 33, n. 2, pp. 1-4.

https://doi.org/10.2307/1249394

Michael, Donald N. (1964). "Speculations on the relation of the computer to individual freedom and the right to privacy". George Washington law review, v. 33, n. 1, pp. 270-286.

Michelfelder, Diane P. (2010). "Philosophy, privacy, and pervasive computing". Al \& society, v. $25, \mathrm{n} .1$, pp. 61-70. https://doi.org/10.1007/s00146-009-0233-2

Min, Jinyoung (2016). "Personal information concerns and provision in social network sites: Interplay between secure preservation and true presentation". Journal of the Association for Information Science and Technology, v. 67, n. 1, pp. 26-42. https://doi.org/10.1002/asi.23376

Moore, Adam D. (2010). Privacy rights. Moral and legal foundations. The Pennsylvania State University Press. ISBN: 9780271036854

Moreno-Becerra, Tabita; Gajardo-León, Constanza; Parra-Ortiz, Elizabeth (2016). "Privacidad: cómo se entiende y se gestiona en Facebook. Estudio de caso de jóvenes chilenos". Revista latina de comunicación social, n. 71, pp. 715-729. https://doi.org/10.4185/rlcs-2016-1117

Morozov, Evgeny (2012). The net delusion: The dark side of Internet. New York: Public Affairs. ISBN: 9781586488741

Morris, Linda; Pharr, Steve (1992). "Invasion of privacy: A dilemma for marketing-research and database technology". Journal of systems management, v. 43, n. 10, pp. 10-11; 30$31 ; 42-44$
Murphy, Richard S. (1996). "Property rights in personal information: An economic defense of privacy". Georgetown Law Journal, v. 84, n. 7, pp. 2381-2417.

Noam, Eli M. (1997). "Privacy and self-regulation: Markets for electronic privacy". En: Wellbery, B.S. (ed.), Privacy and self-regulation in the information age, cap. 1-B. National Telecommunications and Information Administration, Washington.

http://www.ntia.doc.gov/page/chapter-1-theory-marketsand-privacy

Norberg, Patricia A.; Horne, Daniel R.; Horne, David A. (2007). "The privacy paradox: Personal information disclosure intentions versus behaviors". Journal of consumer affairs, v. 41, n. 1, pp. 100-126.

https://doi.org/10.1111/j.1745-6606.2006.00070.x

OCDE (2011). Thirty years after the OECD Privacy guidelines. Organisation for Economic Cooperation and Development. http://www.oecd.org/sti/ieconomy/49710223.pdf

Okazaki, Shintaro; Katsukura, Akihiro; Nishiyama, Mamoru (2007). "How mobile advertising works: The role of trust in improving attitudes and recall". Journal of advertising research, v. 47, n. 2, pp. 165-178.

https://doi.org/10.2501/s0021849907070195

Osatuyi, Babajide (2015). "Personality traits and information privacy concern on social media platforms". Journal of computer information systems, v. 55, n. 4, pp. 11-19. https://doi.org/10.1080/08874417.2015.11645782

Pavlou, Paul A. (2011). "State of the information privacy literature: Where are we now and where should we go?". MIS quarterly, v. 35, n. 4, pp. 977-988.

https://papers.ssrn.com/sol3/papers.cfm?abstract_id=2369375

Peters, Cara; Amato, Christie H.; Hollenbeck, Candice R. (2007). "An exploratory investigation of consumers' perceptions of wireless advertising". Journal of advertising, v. 36, n. 4, pp. 129-145.

https://doi.org/10.2753/joa0091-3367360410

Phelps, Joseph; Nowak, Glen; Ferrell, Elizabeth (2000). "Privacy concerns and consumer willingness to provide personal information". Journal of public policy \& marketing, v. 19 , n. 1, pp. 27-41.

https://doi.org/10.1509/jppm.19.1.27.16941

Pierson, Jo (2012). "Online privacy in social media: A conceptual exploration of empowerment and vulnerability". Communications \& strategies, n. 88, pp. 99-120.

https://papers.ssrn.com/sol3/papers.cfm?abstract_id=2374376

Posner, Richard A. (1978). "An economic theory on privacy". Regulation: AEl journal on government and society, $\mathrm{n}$. May/ June, pp. 19-26.

https://object.cato.org/sites/cato.org/files/serials/files/ regulation/1978/5/v2n3-4.pdf

Posner, Richard A. (1981). "The economics of privacy". The American economic review, v. 71, n. 2, pp. 405-409. https://www.jstor.org/stable/1815754

Prosser, William L. (1960). "Privacy". California law review, v. 48, n. 3 , pp. 383-423.

https:// doi.org/10.2307/3478805 
Rust, Roland T.; Kannan, P. K.; Peng, Na (2002). "The customer economics of Internet privacy". Journal of the Academy of Marketing Science, v. 30, n. 4, pp. 455-464. https://doi.org/10.1177/009207002236917

Ryan, William G. (1967). "Book review - The problem of privacy: Edward Engberg: The spy in the corporate structure and the right to privacy, The World Publishing Company". Business horizons, v. 10, n. 4, p. 106.

https://ideas.repec.org/a/eee/bushor/v10y1967i4p106106a.html

Samuelson, Pamela (2000). "Privacy as intellectual property?". Stanford law review, v. 52, n. 5, pp. 1125-1173. https://doi.org/10.2307/1229511

Schwaig, Kathy S.; Segars, Albert H.; Grover, Varun; Fiedler, Kirk D. (2013). "A model of consumers' perceptions of the invasion of information privacy". Information \& management, v. 50, n. 1, pp. 1-12.

https://doi.org/10.1016/j.im.2012.11.002

Smith, Wendell R. (1956). "Product differentiation and market segmentation as alternative marketing strategies". Journal of marketing, v. 21, n. 1, pp. 3-8.

https://doi.org/10.2307/1247695

Spiekermann, Sarah; Acquisti, Alessandro; Böhme, Rainer; Hui, Kai-Lung (2015). "The challenges of personal data markets and privacy". Electronic markets, v. 25, n. 2, pp. 161-167. https://doi.org/10.1007/s12525-015-0191-0

Steinberger, Peter J. (1999). "Public and private". Political studies, v. 47, n. 2, pp. 292-313.

http://dx.doi.org/10.1111/1467-9248.00201

Stigler, George J. (1980). "An introduction to privacy in economics and politics". The journal of legal studies, v. 9, n. 4, pp. 623-644

http://dx.doi.org/10.1086/467657

Strahilevitz, Lior-Jacob; Kugler, Matthew B. (2016). "Is privacy policy language irrelevant to consumers?". Journal of legal studies, v. 45, n. S2, pp. S69-S95.

https://doi.org/10.1086/689933

Subramanian, Ramesh; Katz, Eddan (2011). "Perspectives on the global flow of information". En: Subramanian, R. y Katz, E. (eds.), The global flow of information: Legal, social, and cultural perspectives, pp. 1-22. New York University Press. https://doi.org/10.18574/nyu/9780814748114.003.0001

Taylor, Curtis R. (2004). “Consumer privacy and the market for customer information". RAND journal of economics, v. 35, n. 4, pp. 631-650.

https://doi.org/10.2307/1593765

Trepte, Sabine; Reinecke, Leonard (2013). "The reciprocal effects of social network site use and the disposition for self-disclosure: A longitudinal study". Computers in human behavior, v. 29, n. 3, pp. 1102-1112.

https://doi.org/10.1016/j.chb.2012.10.002

Tucker, Catherine E. (2012). "The economics of advertising and privacy". International journal of industrial organization, v. 30, n. 3, pp. 326-329.

https://doi.org/10.1016/j.ijindorg.2011.11.004

Varian, Hal R. (1997). "Economic aspects of personal privacy". En: Wellbery, B. S. (ed.). Privacy and self-regulation in the information age, cap. 1-C. Washington: National Telecommunications and Information Administration.

http://www.ntia.doc.gov/page/chapter-1-theory-marketsand-privacy

Westin, Alan F. (1967). Privacy and freedom. New York: Atheneum. ISBN: 9781935439974

Weintraub, Jeff (1997). "The theory and politics of the public/private distinction". En: Weintraub, J. y Kumar, K. (eds.), Public and private in thought and practice, pp. 1-42. Chicago: The University of Chicago Press. ISBN: 9780226886237

Wu, Kuang-Wen; Huang, Shaio-Yan; Yen, David C.; Popova, Irina (2012). "The effect of online privacy policy on consumer privacy concern and trust". Computers in human behavior, v. 28, n. 3, pp. 889-897.

https://doi.org/10.1016/j.chb.2011.12.008

Young, Alyson-Leigh; Quan-Haase, Anabel (2013). “Privacy protection strategies on Facebook: The Internet privacy paradox revisited". Information, communication \& society, v. 16, n. 4, pp. 479-500

https://doi.org/10.1080/1369118x.2013.777757

Zhang, Li-ping (2016). "On strategies of personal information protection in the personalized information service in big data times". ITM Web of conferences, v. 7, art. 03002. https://doi.org/10.1051/itmconf/20160703002

Zhitomirsky-Geffet, Maayan; Bratspiess, Yair (2016). "Professional information disclosure on social networks: The case of Facebook and LinkedIn in Israel". Journal of the Association for Information Science and Technology, v. 67, n. 3, pp. 493-504.

https://doi.org/10.1002/asi.23393

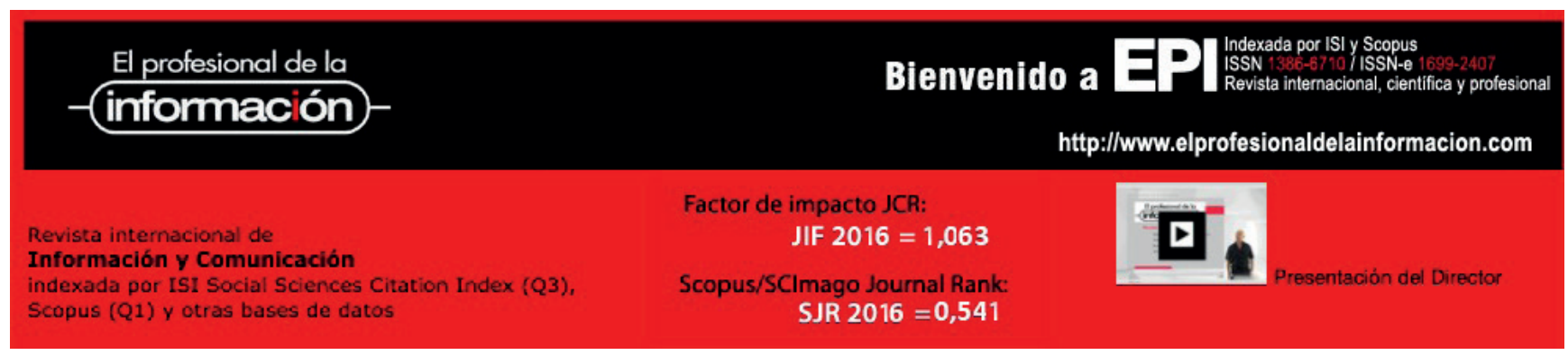

